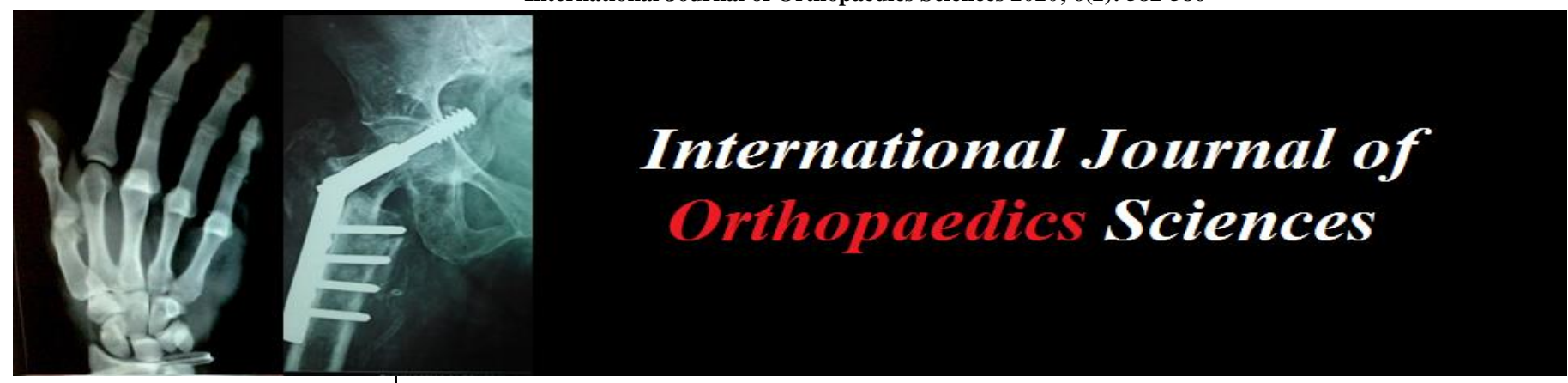

E-ISSN: 2395-1958

P-ISSN: 2706-6630

IJOS 2020; 6(2): 582-586

(C) 2020 IJOS

www.orthopaper.com

Received: 01-02-2020

Accepted: 03-03-2020

Dr. Bindhulal VA

MS Ortho, Additional Professor,

Govt. T.D Medical College

Alappuzha, Kerala University of

Health Sciences (KUHS),

Kerala, India

Dr. Georgekutty

AM MS Ortho, Additional

Professor, Govt. T.D Medical

College Alappuzha, Kerala

University of Health Sciences

(KUHS), Kerala, India

Dr. Alen Sigamani MS

Ortho, Assistant Professor,

Department of Orthopedics

TDMCH, Kerala, India

Dr. Manesh Stephen

DNB (Ortho), Assistant

Professor, Department of

Orthopedics TDMCH, Kerala,

India

Dr. Ahamad Shaheel Sultan

Consultant Orthopaedic Surgeon, Govt. T.D Medical College

Alappuzha, Kerala University of

Health Sciences (KUHS),

Kerala, India

\section{Functional outcome after PFNA osteosynthesis in per trochanteric fractures: A longitutinal study}

\author{
Dr. Bindhulal VA, Dr. Georgekutty, Dr. Alen Sigamani MS, Dr. Manesh \\ Stephen
}

DOI: https://doi.org/10.22271/ortho.2020.v6.i2j.2103

\section{Abstract}

Management of pertrochanteric fractures has been evolving since operative treatment started in orthopedics. The search for ideal implant is a continuous process. This study aims to assess the functional outcome of pertrochanteric fractures following osteosynthesis with PFNA. We followed up 118 cases sub classified into two groups based on Singhs Index with mean follow up of 20 months. The mean age was 68.6 years. The mean operation time was 36.5 minutes (range $24-70$ minutes) and mean blood loss was $220 \mathrm{ml} .57$ patients had varies co morbid conditions which needed special attention and multidisciplinary approach. The overall functional outcome according to Modified Harris Hip score was 90 patients and excellent outcome (score 90-100), 17 patients had good results (score 80-90), 5 had fair outcome (score $70-80$ ) and 6 had poor outcome (Score < 70).102 (87\%) patients returned to prefracture state and were satisfied. We also report 2 rare complications. Overall PFNA gives good to excellent result in $90 \%$ of pertrochanteric fractures and easy to replicate even by a relatively inexperienced surgeon.

Keywords: Per trochanteric fractures, PFNA, DHS, cepallomedullary, AO

\section{Introduction}

Management of pertrochanteric fractures has been evolving since operative treatment started in orthopedics. As the adage goes "man is born through the pelvis and dies because of broken hip bone ", however a lot of advances have been made in the treatment of these fractures primarily due to the work done by the AO group. Massie ${ }^{[1]}$ in his article said operative management of trochanteric fractures began in 1950. ${ }^{[1]}$ Extra medullary implants were first used and the Dynamic hip screw (DHS) became the gold standard, but in unstable fractures implant failure were a common occurrence as emphasized by Davis ${ }^{[2]}$ et al. and thus intramedullary implants were favored more so in unstable fractures ${ }^{[2]}$. Mathew ${ }^{[3]}$ in 2006 mentioned that the first cephallomedullary nailing was done in 1967 and the nail used was Zickel nail which had a slot in the proximal part to enable to pass a screw into neck of femur [3]. The Gamma nail later became popular as developments in designs improved. The earlier designs used single large screws with large diameter but had higher incidence of fractures hence was later replaced by two small diameter screws as mentioned by George K Kouvidis [4]. Thus proximal femoral nail was thus born and it became the favored implant and still continues to hold sway. The problem with the 2 screw design was initially highlighted in series of experimental studies in simulated conditions by Eric Strauss ${ }^{[5]}$ where the investigators demonstrated what they called the $\mathrm{Z}$ affect where the 2 screws migrated. Thus the search for ideal implant that can be used for pertrochanteric fractures, stable and unstable across all age groups continued till the AO group introduced the PFNA or proximal femoral nail antirotation in which compression at fracture site and antirotation is achieved by single helical blade in a slot.

This study was undertaken to assess the functional outcome of pertrochanteric fractures fixed with PFNA above the age of 40 years. We analysed the failure of implant taking into consideration the union of fracture looking for varus collapse and cut through of helical blade which are indicators of poor results. We also sub classified the patient into two groups based on Singhs index into severe osteoporosis with score less than or equal to 3 and another group with score above 3 and hypothesized that the failure rate in patients with severe osteoporosis
Dr. Georgekutty

AM MS Ortho, Additional Professor, Govt. T.D Medical College Alappuzha, Kerala University of Health Sciences (KUHS), Kerala, India 
was likely to be higher as compared to the other group. All patients above the age of 40 with pertrochanteric fractures fixed with PFNA in the study period of 2 years were included in the study. Open fractures were excluded, patients with suspected malignancy or proven malignancy were also excluded. The individual variables like age, sex, medical co morbidities were documented and the Singhs index and other radiological parameters like fracture classification and union, varus collapse, neck shaft angle were noted and verified by two independent observers. On follow up the fracture union clinical and radiological were note and the functional outcome was noted as return to prefracture state.

\section{Method}

On arrival in the emergency room, resusciatation and preliminary assessment was completed. The history noted, xray taken and the classification of fracture according to AO system and the patient was admitted after preliminary investigation with skin traction. The patients we subclassified based on Singh index based on opposite side hip trabecular pattern by two observers. As most cases were elderly patients presurgical work up by a cardiologist, pulmonologist and physician to control diabetic mellitus, hypertension and other co morbidities. After anesthetic clearance, the patient was taken up for elective surgery under spinal and /or epidural. The patient was operated by a senior surgeon on fracture table and image guidance. The nail was passed after reduction and the blade screw hammered in after determining the appropriate length preferably central or slightly inferior in position in the neck, one distal locking bolt was put in all cases. Postoperative management was patient depended, all patients were made to sit up with feet dangling by the side of bed next day. Patients were allowed toe touch walking with walker after suture removal depending on the cognitive function and cooperation of the care givers except in young patient who were allowed toe touch immediately. The patients were followed up on routine bases till union.

\section{Results}

There were total of 129 cases 11 lost to follow up or died hence the total number followed up was 118 over a period of 3 years. Mean follow up was 20 months (range 9 - 36 months). 73 females (Age 49- 86) and 45 males (Age 46 - 92). The mean age was 68.6 years (46 - 92 years) The fractures were classified according to AO classification (Table 1) into various subtypes. The fractures were subclassified into two groups based on Singhs index into osteoporotic less than 3 score and more than 3 score as not osteoporotic (Table 1)

Table 1: The fractures were subclassified into two groups based on Singhs index into osteoporotic less than 3 score and more than 3 score as not osteoporotic

\begin{tabular}{|c|c|c|c|c|c|c|c|}
\hline \multirow{2}{*}{ AO Classification } & \multirow{2}{*}{ NO. } & \multirow{2}{*}{ Male } & \multirow{2}{*}{ Female } & \multicolumn{2}{|c|}{ Singh Index >3 } & \multicolumn{2}{|c|}{ Singh Index < 3 } \\
\cline { 5 - 8 } & & & & $\mathbf{M}$ & $\mathbf{F}$ & $\mathbf{M}$ & $\mathbf{F}$ \\
\hline 31 A1.2 & 20 & 4 & 16 & 4 & 12 & 0 & 4 \\
\hline 31A1.3 & 25 & 7 & 18 & 5 & 10 & 2 & 8 \\
\hline 31A2.2 & 23 & 9 & 14 & 2 & 5 & 7 & 9 \\
\hline $31 \mathrm{~A} 2.3$ & 31 & 16 & 15 & 8 & 5 & 8 & 10 \\
\hline 31A3.1 & 12 & 5 & 7 & 1 & 2 & 4 & 5 \\
\hline 31A3.2 & 3 & 2 & 1 & 2 & 0 & 0 & 1 \\
\hline 31A3.3 & 4 & 2 & 2 & 1 & 1 & 1 & 1 \\
\hline
\end{tabular}

The mean operation time was 36.5 minutes (range $24-70$ minutes) and mean blood loss was $220 \mathrm{ml}$. Out of the 118 patients 105 patients had the blade screw placed central or inferior and 13 patients screw was slightly superior. 8 out of the central or inferior placed blade screw had further migration and 3 had cut out and failure of fixation needing revision. \& 8 out of the 13 patients with superior placement had further superior migration and 3 had cut out and failure of implant. Out of the 118 patients 57 (34 Females \& 23 Males) were hypertensive. 19 of the 57 were on 2 or more antihypertensive drugs. 32 (17 Females \& 15 Males) out of 118 had diabetic mellitus. 27 (12 Females \& 15 Males) were diabetic and hypertensive. 26 patients (11 Females \& 15
Males) had history of coronary artery disease (CAD) with 18 patients on single antiplatelet and 8 patients on dual antiplatelet. Total of 9 patients had history of cerebrovascular accident (CVA). 39 patients had Chronic obstructive pulmonary disease (COPD) and 11 patients out of 39 had severe restriction of $\mathrm{FeV} 1>30 \%$ and 9 patients had chronic kidney disease (CKD) serum creatinine $>1.5 \mathrm{mg} \%$. We had 62 (Female $39 \&$ Males 23) patients with Singhs index less than 3 and 56 (Females $34 \&$ Males 22) more than 3 respectively. It was noted that the 18 patients out of 39 who were diagnosed to have COPD had Singhs Index less than 3 (relative risk RR 0.83 95\% CI 0.46- 0.55).

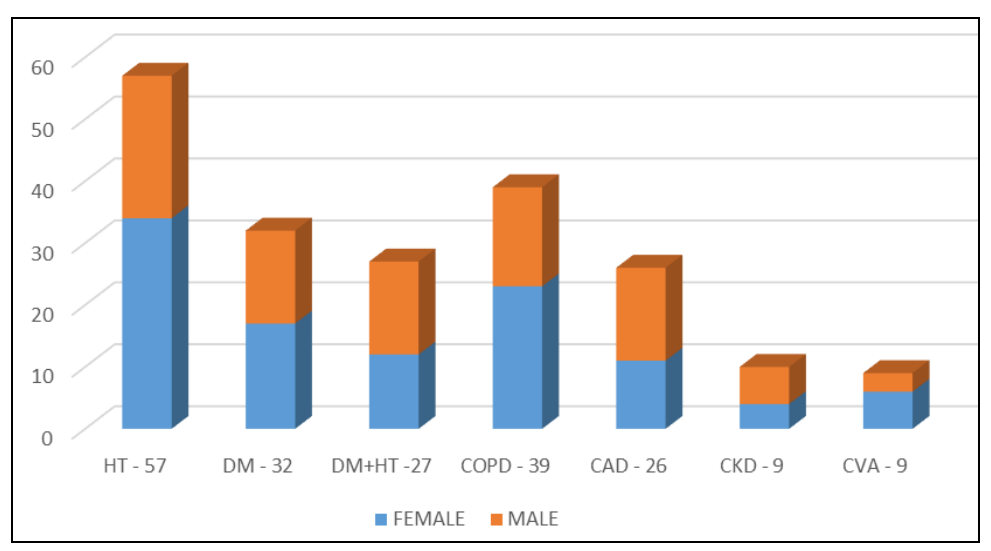

Chart 1: Co -Morbid Diseases $\sim 583 \sim$ 
Out of 118 cases 11 case had varus collapse but 5 of them went on to union and good function while 6 had cut out and failure of implant needing revision. Out of the 56 cases with Singhs index more than 3, 6 cases had varus collapse and 2 had cut out while out of the 62 cases with singhs index less than 3, 7 cases had varus collapse and 4 had cut out with (relative risk RR $1.195 \%$ CI $0.10-0.11$ ). The overall functional outcome according to Modified Harris Hip score was 90 patients and excellent outcome (score 90-100), 17 patients had good results (score 80-90), 5 had fair outcome (score 70-80) and 6 had poor outcome (Score < 70).102 $(87 \%)$ patients returned to prefracture state and were satisfied

\section{Discussion}

Ours is a tertiary level government hospital in south India a referral center. We had total of 118 patients operated for per trochanteric fractures, 73 female and 45 males. Mean age was 68.7 years. Most of our patients come from lower socioeconomic class. To the best of our knowledge there is no published literature on Singhs index in trochanteric fractures and associated complications based on the subclassification into osteoporotic and nonosteoporotic groups. Dual-energy Xray absorptiometry (DEXA) is the gold standard in determining the bone mineral density (BMD) at the hip and spine. But in places were DEXA is not freely available or is relatively expensive as in our case due to the additional cost and logistical problems, singhs index is an alternative tool to assess osteoporosis and subgroup classification and analysis ${ }^{[6]}$. Zhenyu Liu in their paper concluded that a combination of Osteoporosis self - Assessment tool for Asians (OSTA) plus singhs index could be a clinical alternate to assess risk of osteoporosis and associated fractures ${ }^{[6]}$. Osteoporosis is a public health problem particularly in women after menopause, this could be due to hormonal changes associated with menopause poor bone mineral density prior to menopause and inadequate supplementation due to economic condition. Severe osteoporosis leads to hip fractures with minor trauma in elderly and as the adage goes, leads to overall deterioration of health and ultimate demise of patient. Osteoporosis also leads to implant failure due to poor bone stock decreases anchorage, delayed fracture healing, delayed mobilization hence more complications like longer hospital stay and increasing health cost. In the present study there was no significant difference number of patients in both arms 62 osteoporotic and 56 non osteoporotic. But the incidence of comminuted fractures (31 A2.2, 31A2.3 and 31A3.3) were higher in osteoporotic group 34 and 22 respectively (Table 1). This could be due to poor bone quality leading to comminution even with minimal trauma. (RR 1.55 CI 95\% $0.28-0.186)$. Pertrochanteric fractures are essentially fracture of the elderly with mean age of 68.6 as in the present study, hence associated with various co morbidities. 57 (48\%) patients in the present study had one or more lifestyle diseases with hypertension accounting for most number of cases. This could be due to the fact that our hospital is a referral center hence patients with co morbidities may be referred while others may be operated in the periphery. The approach to these patients is multidisciplinary and based on risk to benefit assessment. All diabetic patients were managed with insulin preop and 4 to 6 weeks post op. It was noted that the 18 $(46.15 \%)$ patients out of 39 who were diagnosed to have COPD had Singhs Index less than 3 (relative risk RR 0.83 95\% CI 0.46- 0.55) this is comparable to systematic review by Graat -Verboom ${ }^{[7]}$. Graat- Verboom et al. in a systematic review of 13 studies involving 775 COPD patients reported an overall prevalence of osteoporosis of $35.1 \%$ (range 9-69\%) and osteopenia of $38.4 \%$ (range $27-67 \%$ ). This could be due to poor nutrition, vitamin D deficiency, and poor skeletal muscle function, lack of activity due to poor effort tolerance, hypogonadism, systemic inflammation and chronic steroid use. Placement of blade screw should be ideally central that is the recommendation but there is also another school of thought that states that inferior placement is better in posteromedial comminution that negates the deforming forces in posteromedial comminution and cut out as studied by Lee et al. ${ }^{[8]}$, in our study 105 patients had screws placed central or inferior and 13 slightly superior, 8 out of the 13 placed superior had further migration and 3 cut out. The overall fixation failure rate in trochanteric fractures is $5-8 \%$ according to different studies ${ }^{[9]}$. There were cases of back out of blade screws associated varus collapse Fig 2. Out of the 11 cases with varus collapse 5 went to union with good to excellent (Harris Hip score) function despite neck shaft angle being less than 118 degree, while 6 cases (Fig 3 ) had cut out 2 FIG 2

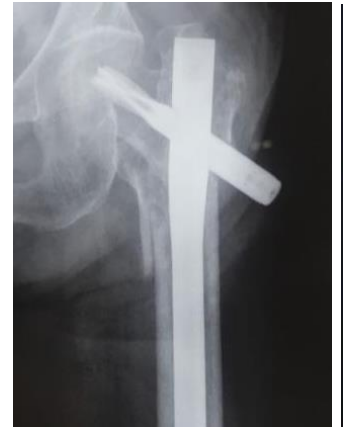

A.

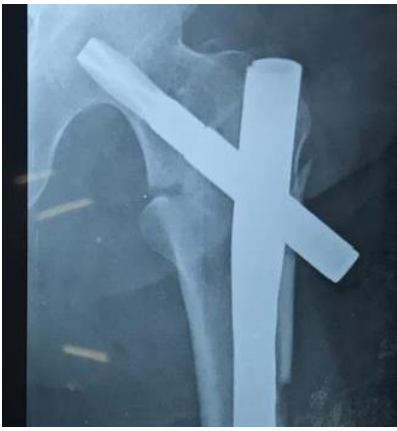

B.

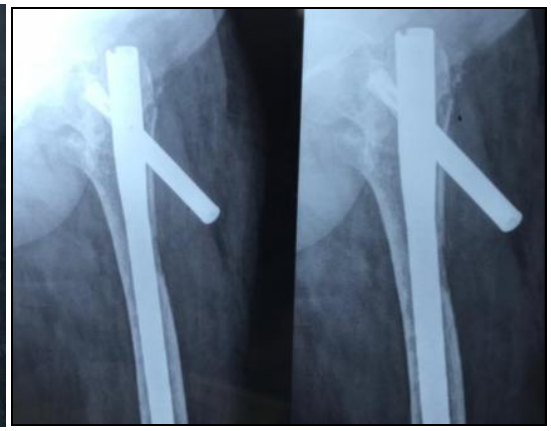

$\mathrm{C}$

Fig 2A: Back out of central placed screw with varus collapse B\& C: Back out of inferior placed screw with varus collapse.

in non-osteoporotic group and 4 in osteoporotic group. Our experience is that the failure rates are more in cases of lateral wall blow out, subtrochanteric extension, coronal split and reverse oblique fractures with proximal extension into greater trochanter. 


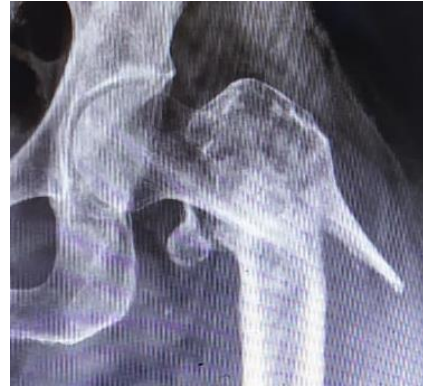

A.

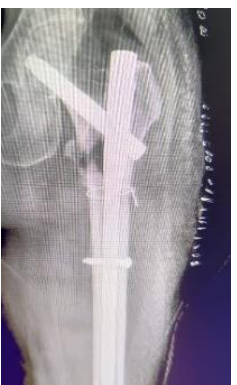

B.

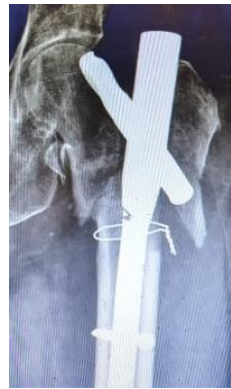

C.

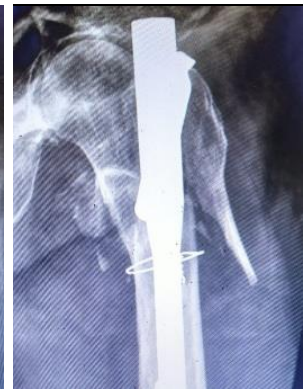

D.

Fig 3: Reverse oblique fracture with lateral wall blow out, anatomical reduction and inferior place blade screw due to posteromedial comminution after wiring, cut out of implant after 4 weeks needing revision with DCS

The overall functional outcome according to Modified Harris Hip score was 90(76.2\%) (Fig 4). patients had excellent outcome (score $90-100), 17(14.4 \%)$ patients had good results (score 80-90), 5(4.2\%) had fair outcome (score 70-80) and $6(5.08 \%)$ had poor outcome (Score < 70).102 (87\%) patients returned to prefracture state and were satisfied. ${ }^{[10,11]}$ This is comparable to the metaanalysis by Zhengan et who in their metaanlysis said the findings support the superiority of PFNA over other treatments for intertrochanteric femoral fracture. PFNA treatment results in the lowest amount of blood loss and the shortest operation time. This study is also comparable to the study by Harshwardhan were the mean age of the patients was 70.83 years of age and mean time of surgery was 38.2 minutes. The mean amount of blood loss in surgery was $110.8 \mathrm{ml}$. In implant related complications, 2 patients had cut out and 1 patient had pull - out of the implant. Functional results according to modified Harris hip scores was found to be excellent in $8(30 \%)$ patients, good in $12(40 \%)$ patients, fair in $7(20 \%)$ patients and poor in $3(10 \%)$ patients. The mean operation time of 36.5 minutes and blood loss of $220 \mathrm{ml}$ is comparable to most metaanalysis ${ }^{[12]}$.
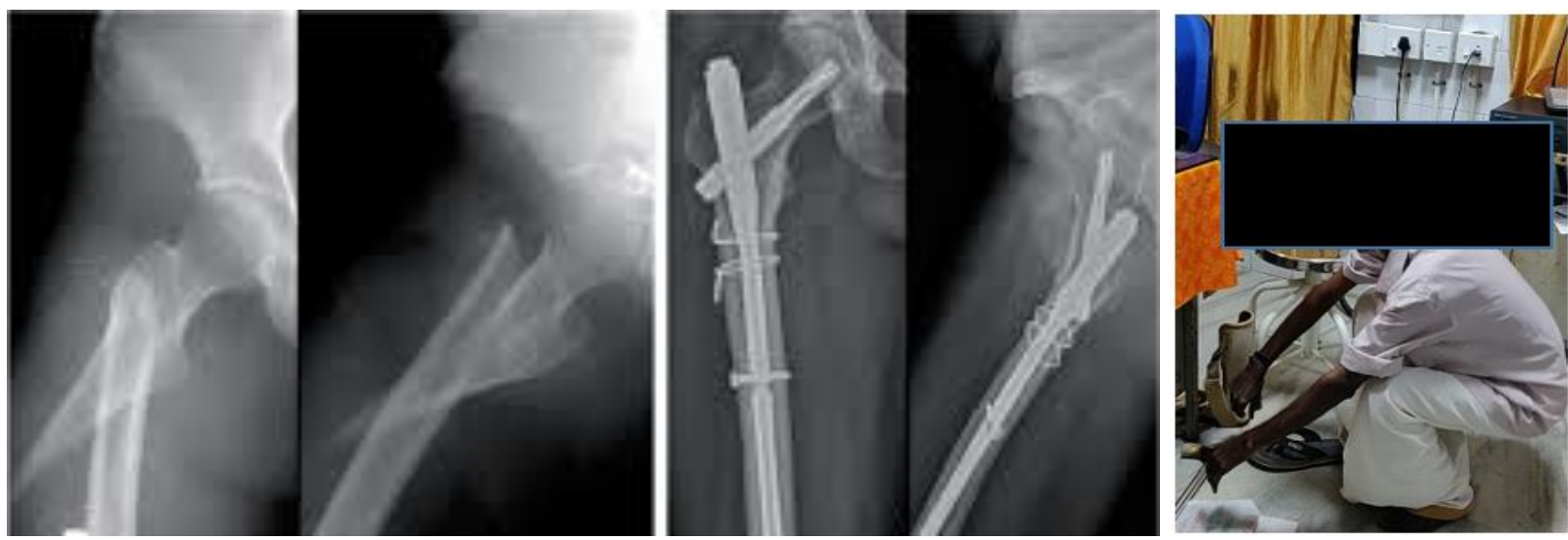

Fig 4: Anatomical reduction, wiring of reverse oblique fracture 31A3.1 and nailing went on to unite and excellent function. Patient able to squat and perform all activities.

Rare complications, we bring to your notice some of the very rare complications not reported in any papers we researched.

Blade screws missing the nail: (Fig:5) this happened in a male patients were the blade screws missed the nail and was not recognized during surgery, but noticed in the immediate post op period and was advised immediate revision, which the patient refused. He had implant failure varus collapse but excellent function with short limp gait could squat and perform all activities and returned to preoperative state.
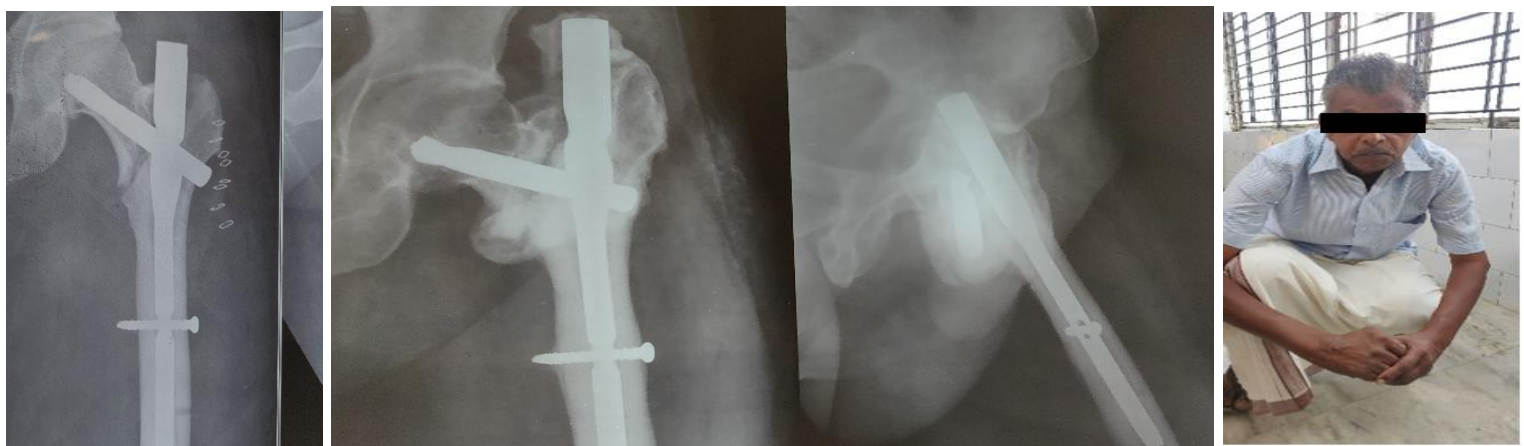

Fig 5: Blade screw missing nail 
Fracture of impaction: (Fig 6) this was noticed during impaction of blade screw in a healthy male of 51 years with Singhs index 6. During impaction we noticed osteochondral fracture of the head. This was noticed in Carm image, no further impaction done the fracture was close to fovea went on to heal uneventfully possibly as it was non weight bearing area

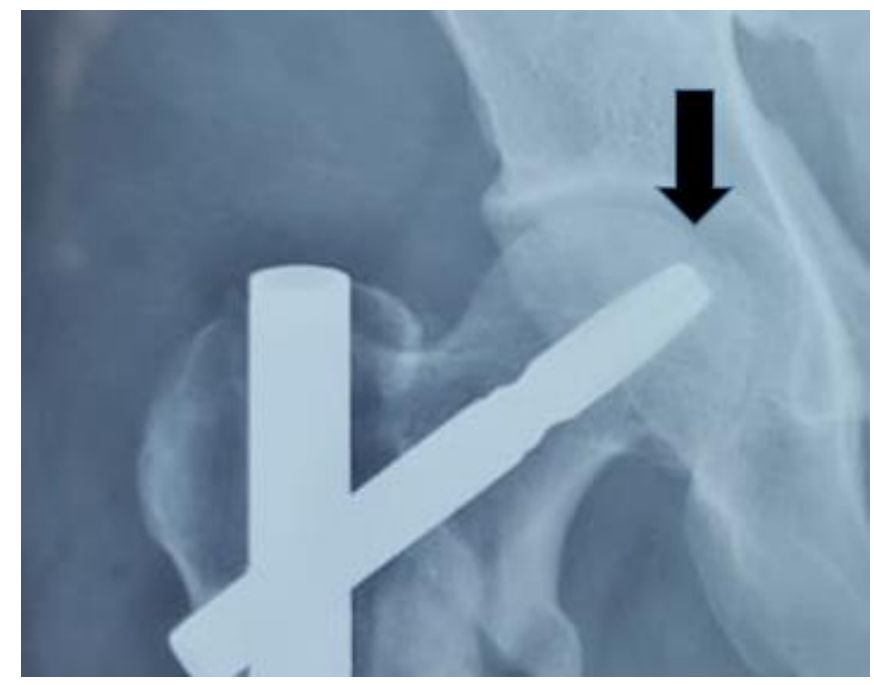

Fig 6: Fracture of impaction close to fovea

\section{Conclusion}

PFNA is a reliable implant in pertrochanteric fracture with good to excellent results in nearly $90 \%$ of patients with very short operating time and blood loss. Easy to replicate even by a relatively inexperienced surgeon after having achieved good reduction. The key point is to achieve anatomical reduction before introducing the nail. It allows early weight bearing in stable fractures and early mobilization in elderly due to minimal post op pain.

\section{References}

1. Massie, William K Fractures of the Hip JBJS. 1964; 46(3):658-690.

2. Davis TR, Sher JL, Horsman A, Simpson M, Porter BB, Checketts RG. Intertrochanteric femoral fractures. Mechanical failure after internal fixation. J Bone Joint Surg Br. 1990; 72(1):26-31.

3. Matthew R, Bong MD, Kenneth J, Koval MD, Kenneth A Egol MD. The History of Intramedullary Nailing. Bulletin of the NYU Hospital for Joint Diseases. 2006; $64(3,4)$

4. George K Kouvidis, Mark B Sommers, Peter V Giannoudis, Pavlos G Katonis, Michael Bottlang. Comparison of migration behavior between single and dual lag screw implants for intertrochanteric fracture fixation; J Orthop Surg Res. 2009; 4:16.

5. Eric Strauss The $Z$ phenomenon A laboratory study Dept. of Orth Surg Inc J. orth Research 25:568-573.

6. Zhenyu Liu, Hua Gao, Xiaodong Bai, Liang Zhao, Yadong Li, Baoju Wang. Evaluation of Singh Index and Osteoporosis Self-Assessment Tool for Asians as risk assessment tools of hip fracture in patients with type 2 diabetes mellitus. J Orthop Surg Res. 2017; 12:37.
7. Graat-Verboom L, Wouters EF, Smeenk FW, van den Borne BE, Lunde R, Spruit MA. Current status of research on osteoporosis in COPD: a systematic review. Eur Respir J. 2009; 34(1):209-18.

8. Lee $\mathrm{CH}, \mathrm{Su} \mathrm{KC}$, Chen KH, Pan CC, Wu YC. Impact of tip-apex distance and femoral head lag screw position on treatment outcomes of unstable intertrochanteric fractures using cephalomedullary nails. J Int Med Res 2018; 46(6):2128-2140.

9. Broderick JM, Bruce-Brand R, Stanley E, Mulhal KJ. Osteoporotic Hip Fractures: The Burden of Fixation Failure. Scientific World Journal. 2013; 2013:515197.

10. Zhengan Hao, Xifeng Wang, Xingqun Zhang. Comparing surgical interventions for intertrochanteric hip fracture by blood loss and operation time: A network metaanalysis. Journal of Orthopaedic Surgery and Research. 2018; 13:157.

11. Hemeshwar Harshwardhan, Shubhanshu Jain, Manish Sharma. An outcome analysis of intertrochanteric fracture of femur managed with proximal femoral nail antirotation II. Inter J Resortho. 2019; 5(4):2455-4510.

12. Zeng C, Wang YR, Wei J, Gao SG, Zhang FJ, Sun ZQ et al. Treatment of trochanteric fractures with proximal femoral nail antirotation or dynamic hip screw systems: A meta-analysis. J Int Med Res. 2012; 40(3):839-51. 